Projets

de paysage

\section{Projets de paysage}

Revue scientifique sur la conception et l'aménagement de l'espace

$8 \mid 2012$

Les concepteurs de jardins et de parcs japonais

\title{
Résurgences paysagères et nouvelles économies agricoles dans le périurbain francilien
}

Programme agriurbain et zone agricole protégée versus opération d'intérêt national

Reinventing landscape and new agricultural economies in the parisian periurban areas

\section{Monique Poulot}

\section{OpenEdition}

\section{Journals}

Édition électronique

URL : http://journals.openedition.org/paysage/15263

DOI : 10.4000/paysage. 15263

ISSN : 1969-6124

Éditeur :

École nationale supérieure du paysage de Versailles-Marseille, Institut national des sciences appliquées Centre Val de Loire - École de la nature et du paysage, École nationale supérieure d'architecture et de paysage de Bordeaux, École nationale supérieure d'architecture et de paysage de Lille, Agrocampus Angers

Référence électronique

Monique Poulot, «Résurgences paysagères et nouvelles économies agricoles dans le périurbain francilien », Projets de paysage [En ligne], 8| 2012, mis en ligne le 14 juillet 2012, consulté le 11 mars 2021. URL : http://journals.openedition.org/paysage/15263; DOI : https://doi.org/10.4000/paysage. 15263

Ce document a été généré automatiquement le 11 mars 2021.

Projets de paysage 


\section{Résurgences paysagères et nouvelles économies agricoles dans le périurbain francilien}

Programme agriurbain et zone agricole protégée versus opération d'intérêt national

Reinventing landscape and new agricultural economies in the parisian periurban areas

Monique Poulot

1 Dans son livre, La Forme d'une ville, le romancier-géographe nantais, Julien Gracq, décrivait les zones périurbaines comme un "véritable chaos où l'on aurait brassé et secoué pêle-mêle les éléments urbains et ceux de la verdure circonvoisine, et où le tout serait resté à l'état d'émulsion mal liée. » (Gracq, 1984.) La formule rend excellemment compte du statut de périphéries dominées, voire de non-lieux (Augé, 1992), attribué aux espaces périurbains. La question périurbaine semble toutefois entrée dans une nouvelle phase et une "stratification » est en train de se faire dans ces espaces habités et revendiqués par plus d'un tiers de la population mondiale ( $25 \%$ en France). En effet, ce périurbain «nomade» (Boino, 1998), au rythme de l'avancée pavillonnaire, s'est engagé dans une démarche de «fabrique à territoires » (Vanier, 1995) : fabrique autour de la figure du «vert» qui le caractérise par rapport à la ville dense, autour des paysages ouverts par opposition aux espaces bâtis, voire autour des espaces agricoles qui représentent une large part de ces espaces ouverts.

2 Cette nouvelle actualité prend acte de configurations urbaines inédites et des « fortes dispositions paysagistes des sociétés occidentales» (Donadieu, 2002) si bien que les aménageurs et édiles sont pressés de penser l'aménagement à des échelles nouvelles incluant ville compacte et « autre ville » (Dubois-Taine et Chalas, 1997), et d'envisager des mises en cohérence de formes d'occupation du sol et d'activités autrefois exclusives. Dans nombre de projets d'urbanistes, « l'image de la ville [...] doit intégrer le non-bâti et la végétation » (Sieverts, 2004, p. 73); la ville est devenue «fertile » selon le 
titre d'une exposition du printemps 2011 à la Cité de l'Architecture et du Patrimoine de Paris. Enfin, cette évolution intervient dans un contexte alimentaire qui conjugue peur des pénuries et de la "mal-bouffe" au point de redonner une place centrale à l'agriculture en périurbain qui, convertie à la multifonctionnalité depuis la nouvelle politique agricole commune de 1992, retrouve une légitimité.

3 Les espaces périurbains sont ainsi devenus des espaces-enjeux pour repenser et la ville et la campagne, expérimentant des innovations tant paysagères qu'urbanistiques, tant économiques que sociales, s'attachant à promouvoir la modalité de l'agriurbain (Vidal et Fleury, 2007). Cet article souhaite interroger les agencements en cours en Île-deFrance, souvent pionnière en la matière et qui aspire au titre de première écorégion européenne. Alors que l'agriurbain semble l'horizon à atteindre pour tous les acteurs, les modalités territoriales de la cohabitation peinent à se mettre en place. Elles soulèvent trois questions qui formeront la trame de l'exposé : celle des échelles ou des territoires d'intervention; celle des acteurs et de leur coalition pour faire advenir une gouvernance multiniveau; celle enfin des pratiques au quotidien pour inventer un aménagement urbain et rural partagé. Une attention particulière sera portée à l'opération Seine-Aval qui figure une sorte de cas d'école ${ }^{1}$.

\section{Le territoire agriurbain en ses lieux : une question d'échelles}

4 Les processus de territorialisation autour des espaces ouverts dans le périurbain montrent des formalisations nombreuses et multiples. Certaines territorialisations sont proclamées " par le haut » dans le cadre des reconfigurations territoriales entamées au début des années 1990 en France et des opérations d'aménagement du territoire. Mais d'autres formes ou territorialisations "par le bas » sont le fait du jeu des différents acteurs, dont les citoyens-habitants-consommateurs et les agriculteurs. Les échelles mises en œuvre ne sont pas les mêmes et le report des décisions échoit aux seules échelles intermédiaires ou grandes avec "tous les petits arrangements entre amis » (Beurret et Trehet, 2001).

\section{Les territoires périurbains : une frange dans les programmes d'aménagement ou la problématique paysagère des entrées de villes}

5 La revendication d'espaces ouverts entretenus par l'agriculture, bref d'un lieu de vie agréable et attractif, s'affirme en priorité dans les espaces périurbains structurés par des éléments paysagers forts, à l'image du cours d'eau. Le cas parisien, site de confluence hydrographique par excellence, est emblématique avec un réseau dense de vallées découpant des lanières de plateaux. Traditionnellement «les céréales [y] couvraient les plateaux calcaires [...] entrecoupés de la verdure des prairies dans les vallées grandes ou petites tandis que les forêts ou les vergers ourlaient les corniches et soulignaient les buttes et les mamelonnements" (Beaujeu-Garnier, 1977, p. 132). Les vallées de la Seine, de l'Oise ou de l'Orge, ont ainsi participé de l'agriculture de banlieue (Phlipponneau, 1956) ou de la ceinture maraîchère francilienne qui a perduré jusqu'à la Seconde Guerre mondiale. Mais ces vallées qui concentrent les voies de communication ont également reçu les grandes infrastructures industrielles et énergétiques et figuré les lieux majeurs d'urbanisation: le parcellaire y était favorable pour un habitat 
pavillonnaire et les petits agriculteurs-propriétaires, âgés et souvent sans successeur, en butte à la concurrence de régions mieux placées, ont aliéné une partie de leur patrimoine (Poulot et Rouyres, 2000). Ces vallées et leurs versants conservent toutefois des traces de l'ancienne occupation viticole, arboricole ou maraîchère : fines parcelles en lanières, lambeaux de vergers ou présence d'arbres isolés... à l'origine d'un paysage particulièrement apprécié par les nouveaux habitants (Poulot, 2008).

Ces vallées qui figurent des voies d'amenées dans la ville dense, ponctuées de villesportes, ont bénéficié d'un traitement particulier dans les schémas d'aménagement successifs de l'île-de-France. Elles fournissent également les grandes lignes de l'architecture du découpage environnemental adopté depuis 1994 qui organise l'île-deFrance en trois auréoles concentriques (ville-centre, ceinture verte, couronne rurale) reliées par les vallées et liaisons vertes (plan vert, 1995) (figure $n^{\circ} 1$ ). Et ces «places » (Lussault, 2009) focalisent aujourd'hui les grands projets structurant la région capitale : avec de vastes portions classées "territoires prioritaires", la Seine accueille depuis 2006 deux opérations d'intérêt national (OIN), Seine-Amont et Seine-Aval. Ces opérations pilotes d'un développement durable affichent des ambitions environnementales et paysagères avec la réhabilitation des berges de Seine et la sauvegarde des espaces ouverts et agricoles dans un souci d'urbanisme renouvelé et de recréation de circuits courts.

Figure 1. Le découpage environnemental de l'île-de-France : un plan vert structuré en couronnes et en vallées

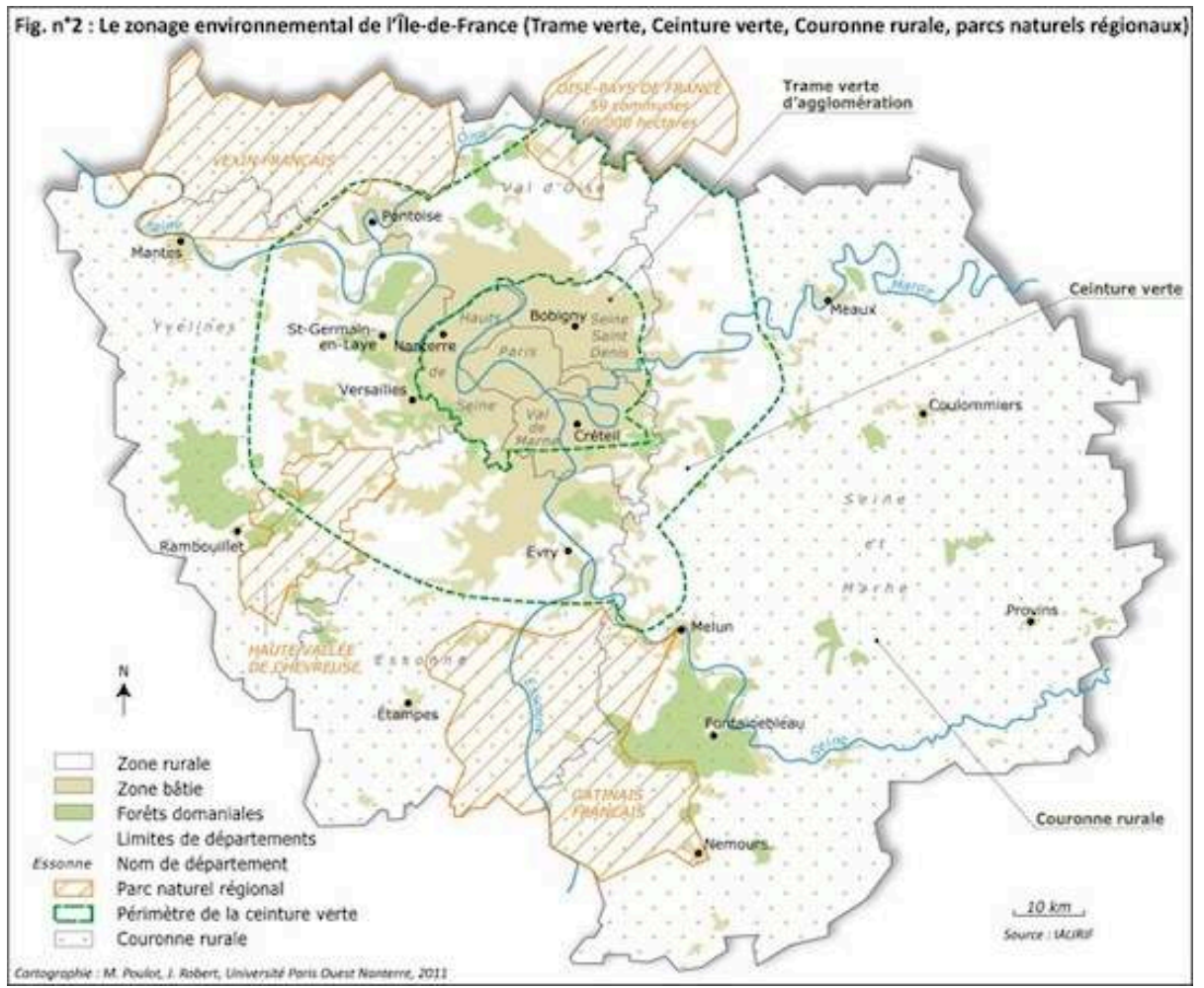

7 L'OIN Seine-Aval regroupe ainsi quelque 300000 habitants sur 51 communes de Conflans-Sainte-Honorine aux portes de l'Eure (figure $n^{\circ} 2$ ) juxtaposant une urbanisation pavillonnaire lâche et quelques centres urbains à forte concentration de logements sociaux (50\% à Mantes-la-Jolie et aux Mureaux et $70 \%$ à Chanteloup-lesVignes). Les contrastes sont marqués entre la vallée à forte tradition industrielle en 
crise $^{2}$ et les versants et plateaux encore largement agricoles ou forestiers. Ce territoire en OIN, sorti de la loi commune à l'image des villes nouvelles, n'est toutefois pas le porteur d'actions agricoles : cette fonction a été dévolue en 2009 à une portion plus restreinte de 16 communes (134 482 habitants), reconnue territoire de Liaison entre actions de développement de l'économie rurale (Leader) (figure $n^{\circ} 2$ ). On est là en présence d'un premier hiatus entre deux territoires de développement portés par l'État et les collectivités locales: d'un côté un ensemble englobant, projet majeur pour la capitale, de l'autre un espace plus restreint financé à $50 \%$ par l'Europe sur des actions ciblées. Quand l'OIN Seine-Aval montre une unité fonctionnelle (polarité logistique et industrielle), le Leader peine à définir un projet agricole et paysager commun entre des communes sans agriculture (jardins familiaux aux Mureaux), celles marquées par la pollution des sols (la boucle de Chanteloup), celles à terroirs multiples (de la vallée au plateau comme Vernouillet), et celles à terroir unique. L'obtention du Leader est d'ailleurs conçue par nombre d'acteurs comme une compensation aux objectifs d'urbanisation massive prévus dans l'OIN.

Figure $n^{\circ} 2$. L'opération d'intérêt national Seine-Aval et le Leader Seine-Aval : problématique des entrées de ville et vision de l'agriculture dans I'OIN

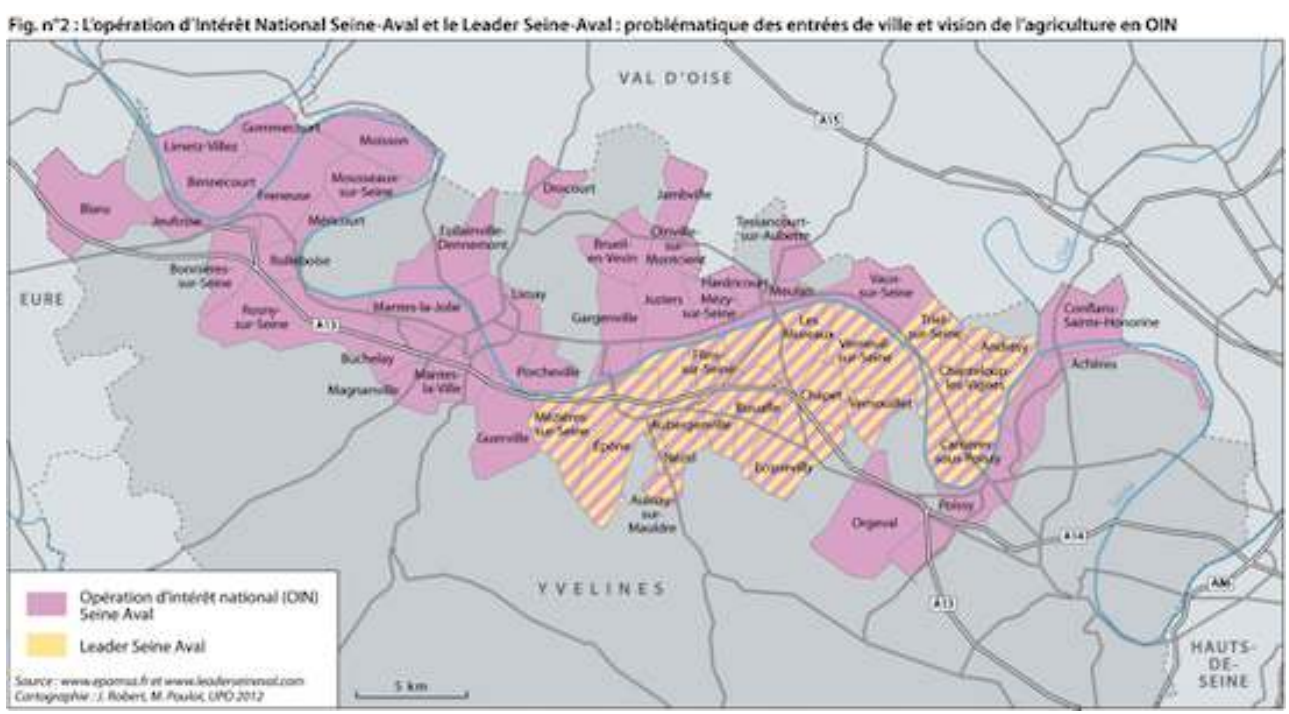

\section{L'agriculture en périurbain : une approche à grande échelle ou la notion d'enclave}

8 Mais les outils de sauvegarde des espaces ouverts convoquent encore d'autres découpages territoriaux. En effet, la Région Île-de-France a opté pour les programmes agriurbains (PAU) ou territoires de projet « dont le périmètre et le contenu sont définis par les partenaires eux-mêmes [...], en réponse à une quête identitaire » (loi rurale, février 2005). Localisés prioritairement en position externe de la ceinture verte, les neuf programmes agriurbains franciliens épousent le front d'urbanisation et dessinent des espaces à forte valeur paysagère ayant déjà connu des interventions de maintien de l'agriculture (Poulot, 2006 et 2011) (figure $n^{\circ}$ 3). 
Figure $n^{\circ} 3$. Les programmes agriurbains en Île-de-France, une localisation préférentielle en ceinture verte et autour de sites à forte valeur paysagère et agricole

Fig. n' 3 : Les programmes agri-urbains en lle-de-France.

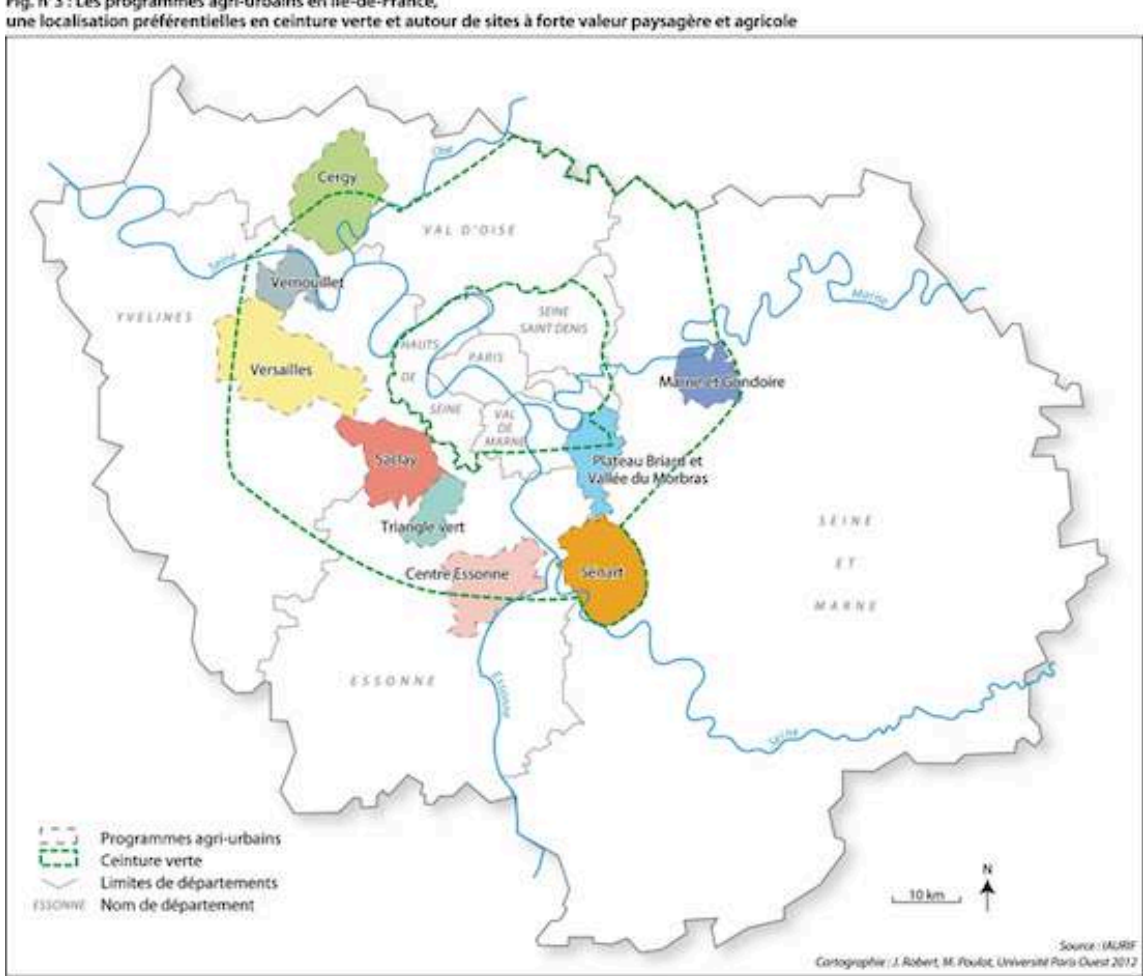

9 Ainsi en Seine-Aval, la Région a institué le programme agriurbain de Vernouillet: inclus en partie dans l'OIN et le Leader, il ne regroupe que huit communes soit 40000 habitants. Ses espaces agricoles, où les cultures spéciales - certaines quasi patrimoniales comme la vigne - côtoient les grandes cultures, sont reconnus par la Région comme "essentiels pour l'image de la capitale » en tant qu'espaces de «transition, entrées ou sorties de ville » (De Biasi et Pujol, 2005) (figure nº 4). Mais alors que le périmètre fonctionnel retenu s'étend sur huit communes, seules deux ont adhéré $^{3}$ : Vernouillet, porteur du projet dès le début des années 2000 et Chapet en 2006. L'espace d'action en faveur de l'agriculture se réduit donc à la seule commune de Vernouillet qui a obtenu en 2008 un arrêté de zone agricole protégée (ZAP), mobilisant un des outils proposés par la loi d'orientation agricole de 1999 (Serrano et Vianey, 2007) (figure $\left.n^{\circ} 4\right)$. 
Figure $n^{\circ} 4$. Le programme agriurbain de Vernouillet : de l'importance des versants de la Seine avec la création d'une ZAP

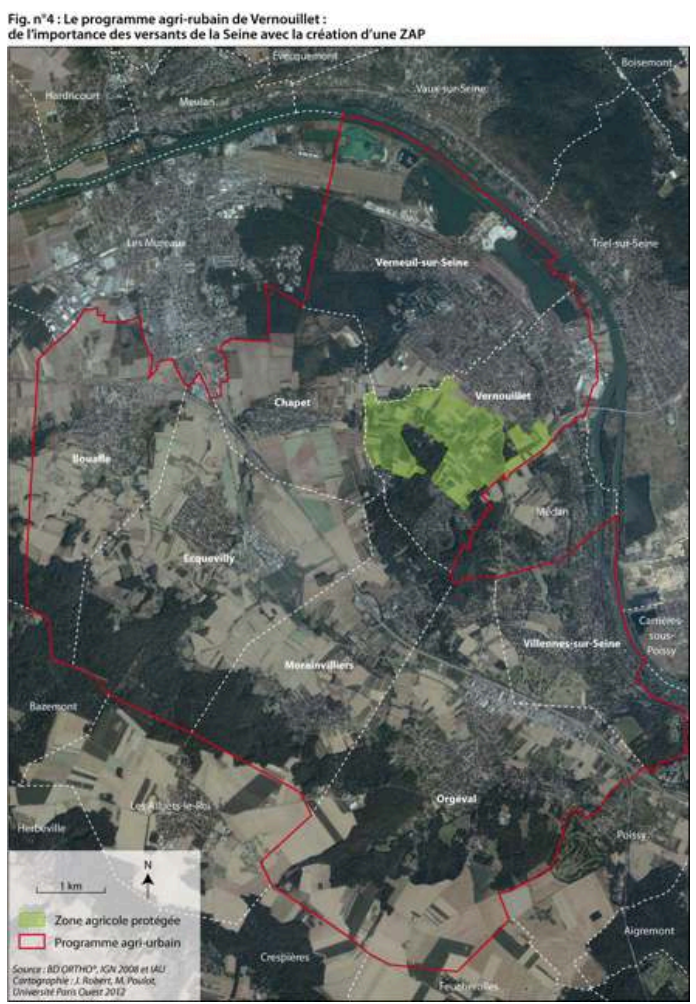

10 Ce feuilletage territorial des procédures soulève la question de l'échelle d'action des projets agriurbains. La taille ${ }^{4}$ en figure une première entrée et la commune de Vernouillet, réduite aux 200 ha de la ZAP, ne peut constituer une échelle pertinente dans une problématique paysagère d'entrée de ville ou dans l'activation de circuits courts à l'échelle de l'OIN. Les périmètres du Leader et du PAU offrent a contrario une taille suffisante mais comment lire l'absence de lien entre ces deux territoires et l'adhésion des communes de Bouafle, Ecquevilly, Orgeval et Verneuil-sur-Seine au Leader après leur refus de participation au PAU : les logiques de guichet l'emportentelles sur les logiques de territoires? Enfin, les horizons de certains agriculteurs dépassent largement les périmètres retenus : les stratégies d'agrandissement, de repli, de diversification ont conduit à des acquisitions ou des locations dans le Vexin normand par exemple, notamment pour les céréaliers, ce qui revient à poser la question des limites d'un territoire agricole d'action. Peut-on l'enfermer dans le territoire d'action de la ville via les documents d'urbanisme?

11 L'inscription territoriale de "terroirs urbains et paysagers " (Larcher, 1998) peine à trouver une échelle d'adhésion et d'action en dépit des nouvelles opportunités des territoires de projets, procédures quasi dérogatoires et sans lien obligé avec les intercommunalités. Cette difficulté témoigne de l'inclusion de l'agriculture et des paysages afférents dans le champ de l'action territoriale urbaine dans le cadre d'enjeux qui à l'évidence ne sont pas qu'agricoles et posent la question des acteurs et de leurs projets. 


\section{Le territoire agriurbain aux prises avec les acteurs: territorialités et temporalités dissociées}

12 En effet, les crises agricoles des dernières années, la décentralisation et la mise en ville de l'agriculture avec la périurbanisation et la réforme des collectivités territoriales ont profondément changé le portage de l'agriculture. Longtemps réduit à un dialogue entre État et profession (Hervieu et Viard, 2001) quand l'agriculture était l'affaire de spécialistes, ce dernier ressortit désormais d'une multitude d'acteurs aux intérêts souvent contradictoires d'où les tensions pour aller vers un projet partagé et une gouvernance associée.

\section{Des territorialités multiples à la recherche d'un bien commun}

13 Si l'agriculture en entrant dans l'arène publique a gagné le statut de bien commun avec pour corollaire la stigmatisation de la consommation effrénée d'espaces ouverts, elle sous-tend dans les discours des territorialités multiples difficilement compatibles (Poulot et Rouyres, 2003).

14 La conversion des édiles aux espaces ouverts est récente et n'intervient qu'à partir d'un certain seuil de constructions neuves et d'apport de nouvelles populations (Charmes, 2011). Relativement forte dans l'Ouest francilien, elle s'appuie sur une vision identitaire et patrimoniale de l'agriculture conçue comme une réserve d'espaces naturels et un ancrage valorisant pour des populations hypermobiles dans un souci de distinction par rapport au centre de l'aire urbaine. Les paysages hérités de la viticulture ou de l'arboriculture des coteaux de Seine sont plébiscités d'autant qu'ils peuvent s'inscrire dans des manifestations culturelles à caractère patrimonial. Mais des oppositions se dessinent entre les communes de vallée urbaines et industrielles et les autres plus agricoles aux terroirs différenciés : dans ces dernières, la ville est rejetée avec une forte valorisation des paysages agricoles et forestiers et du patrimoine architectural ${ }^{5}$.

Le cas de Vernouillet est emblématique de l'évolution des représentations des habitants et des territorialités associées. C'est le projet d'une usine d'incinération des déchets dans la vallée Goujon en 1993 qui déclenche une "crise salutaire " (Darly et Torre, 2008) avec la création de l'Association de défense du cadre de vie de Vernouillet et un changement de majorité municipale. Très Nimby ${ }^{6}$ dans un premier temps, le discours se fait agricole en 1998: l'agriculture devient l'argument pour garantir l'emploi et préserver un cadre de vie agreste et un environnement de qualité tant au niveau social, avec le refus de la mixité, que paysager. Les friches qui ont progressivement colonisé les espaces agricoles (4 exploitations en 2000 contre 16 en 1988) focalisent l'attention, d'autant que certaines ont été acquises par des gens du voyage ${ }^{7}$. L'émergence de l'agriculture comme nouvelle figure territoriale de consensus participe ainsi des "territorialités réflexives" liées "à une production de connaissances sur un sousensemble spatial, à la construction d'un public, à la mise en débat des objectifs de l'action des pouvoirs publics et des devenirs d'un espace » (Melé, 2009).

16 La complexité des enjeux et le repositionnement permanent des acteurs jouent sur « le paysage rural, la couleur de l'agricole, la saveur de l'agricole, mais que reste-t-il de l'agricole?» (Luginbuhl, 1991). De fait, loin d'appréhender la réalité agricole francilienne présente, les intérêts des édiles et des habitants se portent quasi 
exclusivement sur les cultures spéciales à dimension paysagère (l'arboriculture), patrimoniale (la vigne), voire de proximité pour ravitailler les marchés locaux (le maraîchage). Deux légitimités agricoles sont pourtant présentes en Île-de-France depuis plusieurs siècles, la grande culture qui occupe l'essentiel des superficies et les cultures spéciales qui n'ont cessé de régresser. Ainsi, les terres labourables ont connu un fort développement dans la période productiviste au point de couvrir plus de $95 \%$ des superficies en 2000 (Vidal, 2010 ; Poulot 2010).

\section{Le temps des uns et le temps des autres : continuités et discontinuités}

17 Ces territorialités multiples renvoient à des temporalités tout aussi variées et non accordées les unes aux autres. Les différences se lisent dans les pas de temps qui fluctuent selon les projets, avec de surcroît des calendriers qui introduisent nombre de discontinuités.

Le feuilletage des pouvoirs, encore accru dans la région capitale, se marque par des projets devenus vitrines des échéances électorales. Les opérations d'intérêt national, points d'ancrage du Grand Paris, figurent ainsi le projet de l'État avec un souci des voies de communication qui constituent des risques majeurs de déstructuration des espaces naturels et agricoles. Les programmes agriurbains sont portés par la Région qui affiche depuis la décennie des années 1970 une politique de protection des espaces ouverts contre l'État (Poulot et Rouyres, 2003): la non-ratification par l'État du schéma d'aménagement durant trois ans (2008-2011) en témoigne ${ }^{8}$. Souvent captives dans cette rivalité entre l'État et la Région, les communes évoluent selon des temps électoraux encore différents. Et que dire des Leader dont la périodicité est élaborée à l'échelle européenne (2007-2013 pour les projets actuels).

Mais ces temporalités politiques ne représentent qu'une partie de celles des habitants. Les associations ou coalitions, qui viennent répondre à une émotion, disparaissent rapidement ou changent d'objet. L'Association de défense du cadre de vie de Vernouillet créée en 1993 contre une usine d'incinération s'est plus ou moins fondue en 2001 dans l'Association pour le développement de l'agriculture périurbaine (Adapave) à Vernouillet et ses environs qui a soutenu le programme agriurbain et assuré le portage de la zone agricole protégée. Aujourd'hui, l'Adapave est à son tour concurrencée par l'Association de défense des intérêts vernoliens apparue vers 2005 contre un projet de déviation. Associations et politiques se retrouvent enfin dans le Groupement d'action locale (GAL) lié au Leader et fédéré par l'Association pour un développement agricole durable en Seine-Aval. Cette multiplication des associations avec des portages excessivement ciblés rend $a$ fortiori plus complexe l'avènement d'un consensus.

Les agriculteurs, derniers acteurs, représentent les temporalités les plus longues, accordées à la carrière et à la rotation du capital. Permanents de l'histoire, ils sont aussi les moins nombreux, peu disponibles pour participer aux multiples arènes qui décident l'avenir de l'agriculture. Peu soutenus par la structuration traditionnelle de la profession, organisée selon les échelons départementaux et cantonaux qui n'ont plus de signification dans la métropole parisienne, ils ont investi prioritairement l'Adapave puis le GAL qui deviennent les lieux de négociation mais avec une méfiance entre les pionniers (ceux de l'Adapave) et les suiveurs (ceux du GAL). Des tiraillements existent enfin entre les agriculteurs de proximité et les céréaliers, d'autant que le syndicalisme 
traditionnel a privilégié les seconds constitués en lobbies plus aptes à négocier à Bruxelles qu'à l'échelon intercommunal.

21 Le projet agriurbain témoigne de cette multiplication d'acteurs qui revendiquent un droit de regard, voire d'intervention sur l'agriculture, depuis la naissance officielle de la catégorie "agriculture urbaine et périurbaine » à partir de 1996 dans le cadre du nouveau zonage en aires urbaines ( $45 \%$ de l'agriculture française aujourd'hui). La chose agricole est ainsi quasi confisquée par les nouveaux résidents à fort capital social (Berger, 2004). Au pire, l'agriculture devient alibi pour contenir l'urbanisation souvent sans ou contre les agriculteurs; au mieux, l'agriculture trouve une reconnaissance, soumise toutefois à des formes de contrôle. Cette publicisation de l'agriculture et de ses espaces s'impose comme un des fondements des formes partenariales agriculture/ville qui émergent aujourd'hui autour des agglomérations et appelle des figures inédites d'aménagement.

\section{Faire le territoire agriurbain : gestes et pratiques d'un aménagement inédit}

22 En effet, la question est celle de nouvelles formes d'aménagement susceptibles de répondre aux attentes des habitants (une agriculture avec et sous le regard des urbains et pour les urbains) et à celles des agriculteurs (un métier qui les fasse vivre). Quelles peuvent être les adaptations des modalités classiques de l'aménagement rural ? Quelles sont les innovations pour répondre à la problématique urbaine? Quels usages possibles du droit de l'urbanisme?

\section{L'aménagement rural revisité aux portes des villes : « fabriquer de la terre arable »(Brunhes, 1922)}

Les interventions pour maintenir des espaces agricoles aux portes des villes participent d'abord des techniques classiques du «fabriquer de la terre arable » avec la création d'une organisation parcellaire cohérente et efficace. La charte de développement agricole signée en 2001 par l'Adapave, l'État et la commune de Vernouillet, prolongée par l'arrêté de zone agricole protégée en 2008, en est l'expression.

La grande affaire reste celle de la sécurisation foncière d'autant qu'en zone périurbaine la rente foncière est annihilée par la rente urbaine. Le maître d'œuvre n'a pas changé : la Société d'aménagement foncier et d'établissement rural (Safer), créée lors de la révolution agricole productiviste, s'est dotée de nouveaux statuts avec un élargissement de ses prérogatives au périurbain depuis 2005. En charge du marché foncier agricole, avec comme arme ultime la préemption, la Safer intervient pour éviter le changement d'usage des sols et installer des agriculteurs sur des exploitations viables. Elle partage ses prérogatives, en Île-de-France, avec un organisme tout à fait original, l'Agence des espaces verts (AEV) qui, depuis 1976, pratique aussi, pour la Région, veille et acquisition foncières: on recensait ainsi en $2010^{9}$ quelque 77 périmètres régionaux d'intervention foncière (Prif), situés surtout dans la ceinture verte, regroupant quelque 1800 hectares de surface agricole utile.

Ce partenariat se retrouve sur le programme agriurbain de Vernouillet qui a décliné toutes les procédures disponibles de sécurisation foncière. Ainsi, dès 1992, le 
département use du droit de l'urbanisme pour délimiter un "espace naturel sensible $^{10} »$ (ENS) sur 290 hectares dont 210 hectares de terres agricoles. En 2000, l'AEV, au nom de la Région, agrandit l'ENS et le transforme en Prif sur 334 hectares, confiant à la Safer la prospection qui aboutit à l'acquisition de 29 hectares. Enfin, l'arrêté de zone agricole protégée pris en 2008 vient confirmer la vocation agricole « définitive » de ces terrains puisque la ZAP est, aux termes de la loi d'orientation agricole de 1999 et du Code rural, une servitude d'utilité publique annexée aux documents d'urbanisme ${ }^{11}$ (figure $\mathrm{n}^{\circ} 4$ ). Votée alors que la sécurisation était quasiment achevée, la ZAP autorise aussi, par le biais de déclarations d'utilité publique - relevant du droit de l'urbanisme et du droit administratif - à procéder à des expropriations ou expulsions, notamment de gens du voyage, pour rendre effective la vocation agricole. Autant qu'un outil foncier contre la ville, elle devient ici un outil contre des usages des sols mal identifiés par la loi (occupation liée à des activités de ferrailleurs, entreposages divers, etc.)

Mais au-delà de la sécurisation foncière, l'agriurbain appelle la constitution d'un parcellaire efficace dont les modalités émargent surtout au droit rural, s'appuyant sur le Code civil pour dépasser les blocages. Le remembrement, depuis la loi de 1941, demeure la procédure clef : la commune de Vernouillet y a eu recours d'abord en 1986 puis en 2000 dans le cadre du programme agriurbain. Quasi nécessaire dans les vallées qui pratiquaient traditionnellement la petite culture sur des lanières étroites (Charvet et Poulot, 2006), il est devenu obligatoire avec l'abandon de l'agriculture et les partages successoraux qui ont multiplié le nombre de parcelles et de propriétaires. Quelque 1150 parcelles ont été répertoriées dans le Prif de Vernouillet avec comme préalable l'identification des propriétaires. Pour les propriétaires retrouvés, le Code rural offre la procédure L125 qui, en l'absence de mise en valeur durant trois ans, autorise l'exploitation en faire-valoir direct ou la location à un agriculteur : 96 hectares ont ainsi été loués à des agriculteurs locaux. Pour les propriétaires inconnus, l'article $539 \mathrm{du}$ Code civil permet à une commune de s'approprier les biens en l'absence de paiement de la taxe foncière : 7 hectares ont été préemptés par la Safer. Remembrement et travaux connexes ont été menés de pair, notamment le défrichement et le dessouchage ou encore l'aménagement d'accès. Les terres ainsi aménagées ont conforté 4 ou 5 exploitations existantes mais aucune installation n'a été possible. Ces différentes procédures restent néanmoins lourdes et longues: le coup par coup l'a souvent emporté au détriment d'un aménagement concerté qui aurait pu déboucher sur une installation.

$\mathrm{Au}$ vu des outils qui ne cessent de s'étoffer et de transgresser les différents types de droits, la question de la protection foncière ou de l'affectation agricole "définitive " figure toujours un des horizons de toute politique agricole périurbaine. Face à l'étalement urbain, une demande de «mise en défens » reste la réponse de l'agriculture - et de la ville. Elle participe totalement des logiques de séparation entre ville et agriculture qui ont failli déboucher sur la promotion d'une politique d'exception vis-àvis de l'agriculture périurbaine (Larcher, 1998). La création des ZAP en représente un dernier avatar, intervenant quasiment à contre-courant quand la logique de séparation est remise en question. Ces ZAP inscrivent toutefois des points d'ancrage de l'agriculture autour desquels s'organiseront pour les prochaines années et la ville et l'agriculture. Elles requièrent en outre un accord entre les différents partenaires, agriculteurs, propriétaires, chambres consulaires, résidents, usagers, élus, qui autorise connaissance et reconnaissance des uns et des autres: les agriculteurs sont ainsi assurés de l'intérêt des communes qui les abritent. Enfin, elles font entrer l'agriculture 
dans le champ du patrimoine quand les seuls espaces naturels y avaient droit jusqu'alors.

\section{Organiser la cohabitation entre ville et agriculture : résurgences paysagères et nouvelle économie agricole}

La charte de développement agricole signée en 2001 proclamait l'engagement solidaire de tous "à travailler de concert au développement de l'agriculture périurbaine " (articles 1 et 2) puis déclinait les responsabilités individuelles afin d'assurer le succès de cette charte (5 articles). L'invention d'un vivre ensemble agriculture-ville n'a toutefois débuté que récemment, après la restructuration des terres agricoles, relancée par l'obtention du Leader: la priorité est désormais de «créer des liens durables entre mondes agricoles et urbains et le parti pris est d'aider l'agriculture à tirer profit de l'urbanisation grâce aux nouveaux besoins des consommateurs " (GAL Seine-Aval, 2009). Le plan de développement du Leader retient douze actions qui portent spécifiquement sur l'agriculture de proximité et les résurgences paysagères pour une entrée de ville de qualité.

Les contrats en cours de signature entre les agriculteurs et le GAL s'attachent notamment à promouvoir les circuits courts avec, après une étude de marché, l'ouverture d'un point de vente collectif à Vernouillet, qui regroupe la production de douze exploitants. Trois d'entre eux ont bénéficié d'aides financières européennes pour améliorer leur production (acquisition de frigorifiques et couverture antipluie pour verger, aides à la diversification). Cette structure collective complète certaines initiatives individuelles : ainsi le maraîcher vernolitain est aussi le fermier de famille de deux Amap $^{12}$, l'une sur Clamart et l'autre sur Montrouge. Cette nouvelle économie agricole est encore balbutiante mais l'adhésion de nouveaux agriculteurs, hors de Vernouillet, commence à donner forme au projet agriurbain.

Le programme d'actions appelle en effet de ses vœux une agriculture multifonctionnelle : à la fois activité économique, composante de l'armature spatiale et paysagère du territoire autorisant une synthèse entre les campagnes paysage et nature (Perrier-Cornet, 2002) et projet citoyen renouant avec le passé agricole pour donner sens au territoire concerné (Duvernoy et al., 2005). Une attention particulière est portée aux zones de contact entre espaces bâtis et agricoles : l'installation de jardins familiaux ou jardins partagés vient ainsi ménager des transitions avec des clôtures sous forme de haies basses d'aubépines. Des agriculteurs se sont engagés dans une formation pour monter des ateliers pédagogiques d'animation sur l'arboriculture et le maraîchage, tant sur leurs exploitations que dans ces jardins, avec, à terme, la replantation d'une vigne. De surcroît, le GAL soutient l'émergence d'une filière écoconstruction autour de la culture du miscanthus à Chanteloup-les-Vignes: l'objectif est d'empêcher la percolation des polluants dans les sols mais aussi de faire naître un «cœur vert » en entrée de ville au vu des qualités paysagères de cette plante. Enfin, les différents acteurs du GAL envisagent une signalétique sur la ZAP et sur le territoire Leader pour aider à l'appropriation de ces espaces par les habitants et forger une identité visuelle, partie prenante de l'identité globale de ces espaces.

31 C'est au final la question de l'ouverture ou de la cohabitation au quotidien qui est posée par les acteurs du programme agriurbain. Horizon évident dans ce vivre ensemble, sa mise en œuvre reste problématique. Au-delà du pari de la connaissance pour aller vers 
une re-connaissance par le biais des ateliers pédagogiques, les territoires agriurbains sont à la recherche de solutions pour empêcher les dégradations sur les parcelles agricoles. La brigade équestre de surveillance a été abandonnée car peu efficace ; la signalétique tourne parfois aux barrières mobiles et aux fossés; le grand nettoyage de printemps pour évacuer les débris déposés sur les terres agricoles ne peut suffire à pacifier les relations. La nécessaire composition entre les acteurs n'est pas chose évidente d'autant que la ville s'est organisée selon une spécialisation des lieux et une mise à distance de l'agriculture. À l'évidence, l'entrée de l'agriculture dans l'ère de la publicisation (Le Caro, 2007) ressortit tout autant d'une réinvention de la ville que de celle de l'agriculture et appelle une réflexion sur l'espace public et les modalités de réalisation de ces espaces, qu'ils soient paysagers ou monumentaux.

\section{Conclusion}

L'agriurbain qui se met en place en île-de-France, mais aussi dans toutes les grandes métropoles, montre une volonté certaine d'organisation des «éléments urbains et ceux de la verdure circonvoisine " (Gracq, 1984). La difficile stratification rend compte d'un long passé d'exclusion de l'un par l'autre, à tout le moins d'une tolérance a minima dans des espaces confinés (Poulot, 2011). La nouveauté réside dans la reconnaissance mutuelle d'un possible enrichissement, voire d'une nécessaire hybridation de l'un par l'autre : loin de penser l'agriculture contre la ville, il convient de penser l'agriculture articulée avec la ville. L'évolution de la problématique des entrées de villes en porte témoignage: longtemps préemptée par l'aménagement urbain, elle appelle des solutions agricoles et conjugue désormais aménagement rural et urbain. Certes, les tâtonnements sont nombreux dans les manières de faire mais la promotion de la multifonctionnalité, de l'agriculture et des espaces qui la portent, offre des opportunités. L'agriculture dans la ville n'a d'autre choix que d'être multifonctionnelle, cultivant notamment à côté de son incontournable volet production son pouvoir de création d'aménités et celui d'identification. L'articulation des paysages agricoles avec l'urbain ouvre ainsi la voie à un aménagement encore en invention, l'agriurbanisme qui se doit aussi de lutter contre une vision urbaine bien réductrice de l'agriculture, niant notamment la grande culture. Le programme de la Seine-Aval n'échappe pas à ce prisme étroit qui, au moins en Île-de-France, ne peut rendre compte de l'agriculture locale et des paysages qu'elle a forgés sur la longue durée. Ces territoires, s'ils veulent promouvoir un nouveau contrat entre l'agriculture et la ville, devront s'ouvrir aussi aux autres composantes de l'agriculture francilienne.

\section{BIBLIOGRAPHIE}

Augé, M., Les Non-Lieux. Introduction à une anthropologie de la surmodernité, Paris, Seuil, coll. « La librairie du Xxe siècle », 1992.

Beaujeu-Garnier, J., Paris et la Région Île-de-France, Paris, Flammarion, 1977, t. I. 
Beuret, J.-E., Trehet, C., « Des médiations pour la gestion de l'espace rural », Le Courrier de l'environnement de l'Inra, $\mathrm{n}^{\circ}$ 43, 2001.

Berger, M., Les Périurbains de Paris. De la ville dense à la métropole éclatée ?, Paris, Éditions du CNRS, coll. « Espaces et milieux », 2004.

Boino, P., « De l'agglomération à la région urbaine : trente ans de changements spatiofonctionnels dans la région urbaine de Lyon ", dans Vanier, M., Scherrer, F., Boino, P., Bourdin, V., Gallo, E., Maiga, M., La conurbanisation : nouvelles périphéries et précarité de l'emploi, rapport de recherche pour la Datar et le Plan urbain, 1998.

Brunhes, J., La Géographie humaine, Paris, Alcan, 1922.

Charmes, E, La Ville émiettée. Essai sur la clubbisation de la vie urbaine, Paris, PUF, coll. « La ville en débat », 2011.

Charvet, J.-P., Poulot, M., « Conserver des espaces ouverts dans la métropole éclatée : le cas de l'Île-de-France ", dans Dorier-Apprill (sous la dir. de), Ville et Environnement, Paris, Sedes, 2006.

Darly, S., Torre, A., « Conflits liés aux périmètres agricoles et périmètres de gouvernance en îlede-France », Géocarrefour, vol. 83, $\mathrm{n}^{\circ}$ 4, 2008.

De Biasi, L., Pujol, D., Les programmes agriurbains : un partenariat entre agriculteurs et collectivités, un nouveau mode de gouvernance, Iaurif-Driaf, 2005.

Donadieu, P., La Société paysagiste, Arles-Versailles, Actes Sud-ENSP, 2002.

Dubois-Taine, G, Chalas, Y. (sous la dir. de), La Ville émergente, La Tour d'Aigues, Éditions de l'Aube, 1997.

Duvernoy, I., Jarrige, F., Moustier, P., Serrano, J., « Une agriculture multifonctionnelle dans le projet urbain : Quelle reconnaissance, quelle gouvernance », Les Cahiers de la multifonctionnalité, $\mathrm{n}^{\circ} 8,2005$.

Gracq, J., La Forme d'une ville, Paris, José Corti, 1984.

Hervieu, B., Viard, J., L'Archipel paysan : la fin de la république agricole, La Tour d'Aigues, Éditions de l'Aube, 2001.

Larcher, G., Les Terroirs urbains et paysagers : pour un nouvel équilibre des espaces périurbains, Paris, Les rapports du Sénat, $\mathrm{n}^{\circ}$ 415, 1997-1998.

Le Caro, Y., Les Loisirs en espace agricole. L'expérience d'un espace partagé, Rennes, PUR, 2007.

Luginbühl, Y., « Le paysage rural, la couleur de l'agricole, la saveur de l'agricole, mais que reste-til de l'agricole ?», Études rurales. De l'agricole au paysage, n 121-124, 1991.

Lussault, M., De la lutte des classes à la lutte des places, Paris, Grasset, 2008.

Melé, P., « Identifier un régime de territorialité réflexive », dans Vanier, M. (sous la dir. de), Territoires, territorialité, territorialisation, controverses et perspectives, Rennes, PUR, 2009.

Perrier-Cornet, P. (sous la dir. de), Repenser les campagnes, La Tour d'Aigues, Éditions de l'AubeDatar, 2002.

Phlipponneau, M., La Vie rurale de la banlieue parisienne, étude de géographie humaine, Paris, Armand Colin, 1956.

Plan vert régional d'île-de-France, Paris, Iaurif, octobre 1995. 
Poulot, M., « Des arrangements autour de l'agriculture en périurbain : du lotissement agricole au projet de territoire ", Vertigo, La revue électronique en sciences de l'environnement, vol. 11, $\mathrm{n}^{\circ}$ 2, 2011, http://vertigo.revues.org/11188.

Poulot, M., «L'agriculture francilienne dans la seconde moitié du Xx siècle : Vers un postproductivisme de proximité ?» Pour, 205-206, juillet 2010.

Poulot, M., « Les territoires périurbains : "fin de partie" pour la géographie rurale nouvelles perspectives? ", Géocarrefour, n 4 , vol. 83, numéro spécial « Géographie(s) rurale(s) en question », 2008.

Poulot, M., « Les programmes agriurbains en Île-de-France : de la "fabrique" des territoires périurbains », actes du colloque « La dynamique des territoires en milieu périurbain et le patrimoine naturel et culturel », Montréal, Réseau Villes Régions Mondes, 2006.

Poulot, M., Rouyres, T., « Les espaces ouverts en Île-de-France : quels enjeux pour quels acteurs? ", Canadian Journal of Regional Science, Revue canadienne de sciences régionales, $\mathrm{n}^{\circ} 2$ et 3 , t. XXVI, été-automne 2003.

Poulot, M., Rouyres, T., « La ceinture maraîchère et horticole francilienne entre production économique et production de paysage ", Méditerranée, $\mathrm{n}^{\circ}$ 3-4, 2000.

Roux, E., Vanier, M., La Périurbanisation : problématiques et perspectives, Paris, La Documentation française, Coll. « Travaux », 2008.

Serrano, J., Vianey, G., « Les zones agricoles protégées : figer de l'espace agricole pour un projet agricole ou organiser le territoire pour un projet urbain », Géographie, Économie, Société, nº 4, 2007.

Sieverts, T., Entre-ville : une lecture de la Zwischenstadt, Marseille, Éditions Parenthèses, coll.

«Eupalinos », 2004.

Vanier, M., « La petite fabrique des territoires en Rhône-Alpes ", Revue de géographie de Lyon, $\mathrm{n}^{\circ} 2$, vol. 70, 1995

Vidal, R., « Territoires ruraux franciliens et paysage », Pour, nº 205-206, juillet 2010.

Vidal, R., Fleury, A., « De la ville nature à la ville agriculture : le projet agriurbain », Anthos, $\mathrm{n}^{\circ} 3$, 2007.

\section{NOTES}

1. Enquêtes menées durant deux ans (2009-2010) par des étudiants de l'université de Paris Ouest Nanterre La

Défense. Bello, V., «Un programme agriurbain à Vernouillet: vers une agriculture intégrée au projet urbain?", master 2, 2010, $160 \mathrm{p}$.

2. Le rapport emplois/actifs est de 0,67 , inférieur à la moyenne départementale et à la moyenne régionale $(0,92)$. Le chômage est important et le phénomène de navettes ne cesse de prendre de l'ampleur.

3. Quatre ont conclu une convention de veille foncière avec la Safer.

4. Cette question de taille ne se mesure pas seulement en hectares ou en nombre d'exploitations mais appelle aussi la prise en compte de considérations économiques et sociales (un réel tissu agricole et d'industries agroalimentaires).

5. Témoignages d'habitants «Avant j'habitais aux Mureaux, j'en suis parti et ce n'est pas pour retrouver ici la banlieue et la même population » ou « avec l'urbanisation continue, on n'a plus la même culture » (Bello, op.cit., p. 112). 
6. Not In My Back Ysard.

7. Ces «terrains familiaux » constituent une terre d'attache pour une famille élargie qui possède ainsi une adresse, scolarise les enfants et se déplace selon des temporalités complexes. Une cinquantaine de familles (600 à 800 personnes) sont installées à Vernouillet et Chapet.

8. Ce blocage interdisait toute modification des documents d'urbanisme aux échelles communales et intercommunales. Un accord est intervenu en mai 2011 avec une mise en révision du schéma et la possibilité de modifications dès lors qu'elles sont non contraires à la loi sur le Grand Paris du 3 juin 2010.

9. Les Prif recouvrent 16000 hectares dont la plupart sont des superficies boisées. L'intervention sur les terres arables est plus récente et participe d'une nouvelle politique régionale.

10. Loi de 1959 modifiée en 1985 qui institue le département en maître d'œuvre avec la mise en œuvre de la taxe départementale des ENS : l'ENS est une procédure du droit de l'urbanisme qui vise à ouvrir dans un délai de dix ans maximum l'espace préempté par le Conseil général au public pour des activités de nature.

11. En ZAP, le changement d'affectation agricole est soumis au régime des autorisations relevant du Code de l'urbanisme ou à l'avis de la chambre d'agriculture et de la commission départementale d'orientation de l'agriculture.

12. Association pour le maintien de l'agriculture paysanne.

\section{RÉSUMÉS}

Les enjeux paysagers et agricoles sont devenus prégnants dans l'agencement des espaces d'une grande agglomération comme l'î̂e-de-France à tel point que les nouvelles procédures, comme les zones agricoles protégées, s'invitent dans les opérations d'aménagement comme les OIN. De telles évolutions illustrent une volonté nouvelle d'un aménagement rural et urbain partagé avec, du côté rural, le maintien d'un paysage patrimonial susceptible de formaliser une entrée de ville de qualité et le souci d'une alimentation saine et de proximité. Le difficile avènement de l'agriurbain renvoie toutefois à trois questions : celle des échelles ou des territoires d'intervention; celle des acteurs et de leur coalition pour faire advenir une gouvernance multiniveau; celle enfin des pratiques au quotidien, autrement dit des formes d'intervention sur les paysages et de leur publicisation.

Landscape and agricultural challenges have became so important into the space organization of a great metropolis such as Ile-de-France that new procedures, as the protected agricultural areas are now included in the planning urban actions. Such evolutions show a new will of a shared rural and urban planning, with, in the rural side, the preservation of a heritage landscape which is likely to formalise a high quality gateway to the city and the concern for a local and healthy food supply. The difficult invention of agri-urban raises three questions : a fisrt question about scales or the intervention areas; a second one about the actors and their coalition; a last one about the everyday practices on landscapes and their publicization. 
INDEX

Mots-clés : entrée de ville, espaces ouverts, territoire agriurbain, zonage, gouvernance

Keywords : gateway to the city, open spaces, agri-urban area, zoning, governance

\section{AUTEUR}

\section{MONIQUE POULOT}

Professeure de géographie, Mosaïques-LAVUE (UMR CNRS 7218), université Paris Ouest Nanterre La Défense.

mpoulotmoreau[at]u-paris10[dot]fr 